\title{
Thermodynamische Betrachtungen von Schmelzdiagrammen einiger Systeme aus Methylchlorsilanen und Lutidinen
}

\author{
Karl Hensen und Michael Dräbing \\ Institut für Physikalische und Theoretische Chemie, \\ Johann Wolfgang Goethe-Universität, Frankfurt am Main
}

Z. Naturforsch. 45a, 1158-1164 (1990); eingegangen am 1. Juni 1990

Thermodynamic Interpretation of the Phase Diagrams of some Methylchlorsilanes with Lutidines

A thermodynamic interpretation of the liquidus curves of some methyltrichloro- respectively dimethyldichlorosilane/lutidine-systems is given. Based on certain approximations it was possible to fit thermodynamic functions to the experimental results to obtain the excess datas of binary systems. A comparison between the models of Redlich-Kister, Wilson and the NRTL-formalism for the free excess enthalpy of binary mixtures is given.

Key words: Phase diagrams, Methylchlorosilanes, Lutidines, Addition compounds, Thermodynamic excess functions.

\section{Einleitung}

Durch die Aufnahme von Schmelzdiagrammen gelang Hensen und Mitarbeitern der Nachweis von Additionsverbindungen zwischen Methylhalogensilanen und organischen Stickstoffbasen bei tiefen Temperaturen [1-8]. Dabei konnte neben den für diese Substanzklasse bekannten 1:1- und 1:2-Verbindungen erstmals die Existenz von inkongruent schmelzenden 2:1-Addukten der Zusammensetzung $\left(\mathrm{MeSiCl}_{3}\right)_{2} \cdot 3.4$ Lutidin, $\left(\mathrm{MeSiCl}_{3}\right)_{2} \cdot 3.5$-Lutidin bzw. $\left(\mathrm{Me}_{2} \mathrm{SiCl}_{2}\right)_{2} \cdot 3.4$ Lutidin aufgezeigt werden $[3,5]$.

Mit Hilfe eines differentiellen Thermoanalysesystems der Firma Mettler (DSC 30) konnten für die Systeme aus Methyltrichlor- und Dimethyldichlorsilan mit Lutidin die Schmelzenthalpien der gefundenen, meist inkongruent schmelzenden Verbindungen, ermittelt werden [3-5].

In der vorliegenden Arbeit wird die thermodynamische Berechnung der Exzeßgrößen aus den Phasendiagrammen von Systemen aus Methyltrichlor- bzw. Dimethyldichlorsilan und den 6 isomeren Lutidinen mittels der Modellansätze nach Redlich-Kister [9], Wilson [10] sowie mit dem Non Random Two Liquid (NRTL)-Ansatz [12] beschrieben. Die in den Parametersätzen für die jeweiligen Ansätze auftretende Schmelz-

Sonderdruckanforderungen an Prof. Dr. Karl Hensen, Institut für Physikalische und Theoretische Chemie, JohannWolfgang-Goethe-Universität, Niederurseler Hang, D-6000 Frankfurt am Main. enthalpie $\Delta H_{\mathrm{S}}$ wird mit den gemessenen Schmelzenthalpien der auftretenden Verbindungen verglichen.

Darüber hinaus werden aus den gemessenen Schmelzenthalpien näherungsweise Dissoziationsenthalpien für die in diesen Systemen auftretenden Addukte berechnet.

\section{Thermodynamische Interpretation der Schmelzdiagramme}

\section{Redlich und Kister-Ansatz}

Nach Redlich und Kister [9] wird für die molare freie Exzeßenthalpie $\Delta G^{\mathrm{E}}$ einer binären Mischphase die Potenzreihe

$\Delta G^{\mathrm{E}}=x_{\mathrm{B}} x_{\mathrm{A}}\left(A+B\left(2 x_{\mathrm{B}}-1\right)+C\left(2 x_{\mathrm{B}}-1\right)^{2}+\ldots\right)$

angesetzt. Die Parameter $A, B, C$ sind empirische, von Druck und Temperatur abhängige Größen. Für die durchgeführten Rechnungen wurde jedoch nur der Parameter $A$ mit $A=a+b T$ als temperaturabhängig angenommen. $B$ wurde als konstant, alle weiteren Parameter Null gesetzt.

Zur Beschreibung der Gleichgewichtskurven der Amine sowie der 1:1- bzw. 1:2-Verbindungen mit Hilfe des Ansatzes nach Redlich und Kister wurden die von J. Gaede [2] aufgrund von Näherungsannahmen aufgestellten Gleichungen verwendet $\left(\Delta H_{\mathrm{SB}}=\right.$ Schmelzenthalpie der reinen Base; $\Delta H_{\mathrm{SAB}}, \Delta H_{\mathrm{SAB}_{2}}$, $\Delta H_{\mathrm{SA}_{2} \mathrm{~B}}=$ Schmelzenthalpien der Verbindungen $\mathrm{AB}$, $\mathrm{AB}_{2}, \mathrm{~A}_{2} \mathrm{~B} ; x_{\mathrm{A}}, x_{\mathrm{B}}=$ Molenbruch des reinen Silans bzw.

0932-0784 / $90 / 0900-1158 \$ 01.30 / 0$. - Please order a reprint rather than making your own copy.

This work has been digitalized and published in 2013 by Verlag Zeitschrift für Naturforschung in cooperation with the Max Planck Society for the Advancement of Science under a Creative Commons Attribution-NoDerivs 3.0 Germany License. 
der reinen Base; $T_{\mathrm{B}}^{\circ}, T_{\mathrm{AB}}^{\circ}, T_{\mathrm{AB}_{2}}^{\circ}, T_{\mathrm{A}_{2} \mathrm{~B}}^{\circ}=$ Schmelzpunkte (in K) der reinen Komponente $B$ bzw. der Verbindungen): Liquiduskurve von B:

$$
T=\frac{\Delta H_{\mathrm{SB}}+\left(1-x_{\mathrm{B}}\right)^{2}\left(a+B\left(4 x_{\mathrm{B}}-1\right)\right)}{\frac{\Delta H_{\mathrm{SB}}}{T_{\mathrm{B}}^{\circ}}-R \ln x_{\mathrm{B}}-b\left(1-x_{\mathrm{B}}\right)^{2}} ;
$$

Liquiduskurve von $\mathrm{AB}$ :

$$
T=\frac{\Delta H_{\mathrm{SAB}}+a\left(1-2 x_{\mathrm{B}}+2 x_{\mathrm{B}}^{2}\right)+B\left(6 x_{\mathrm{B}}-12 x_{\mathrm{B}}^{2}+8 x_{\mathrm{B}}^{3}-1\right)}{\frac{\Delta H_{\mathrm{SAB}}+0,5\left(a+b T_{\mathrm{AB}}^{\circ}\right)}{T_{\mathrm{AB}}^{\circ}}-R \ln \left(4 x_{\mathrm{B}}\left(1-x_{\mathrm{B}}\right)\right)-b\left(1-2 x_{\mathrm{B}}+2 x_{\mathrm{B}}^{2}\right)} ;
$$

Liquiduskurve von $\mathrm{AB}_{2}$ :

$$
T=\frac{\Delta H_{\mathrm{SAB}_{2}}+a\left(2-4 x_{\mathrm{B}}+3 x_{\mathrm{B}}^{2}\right)+B\left(12 x_{\mathrm{B}}-21 x_{\mathrm{B}}^{2}+12 x_{\mathrm{B}}^{3}-2\right)}{\frac{\Delta H_{\mathrm{SAB}_{2}}+(2 / 3)\left(a+b T_{\mathrm{AB}_{2}}^{\circ}\right)+(2 / 9) B}{T_{\mathrm{AB}_{2}}^{\circ}}-R \ln \left((27 / 4) x_{\mathrm{B}}^{2}\left(1-x_{\mathrm{B}}\right)\right)-b\left(2-4 x_{\mathrm{B}}+3 x_{\mathrm{B}}^{2}\right)} ;
$$

Liquiduskurve von $\mathrm{A}_{2} \mathrm{~B}$ :

$$
T=\frac{\Delta H_{\mathrm{SA}_{2} \mathrm{~B}}+a\left(1-2 x_{\mathrm{B}}+3 x_{\mathrm{B}}^{2}\right)+B\left(6 x_{\mathrm{B}}-15 x_{\mathrm{B}}^{2}+12 x_{\mathrm{B}}^{3}-1\right)}{\frac{\Delta H_{\mathrm{SA}_{2} \mathrm{~B}}+(2 / 3)\left(a+b T_{\mathrm{A}_{2} \mathrm{~B}}^{\circ}\right)-(2 / 9) B}{T_{\mathrm{A}_{2} \mathrm{~B}}^{\circ}}-R \ln \left((27 / 4) x_{\mathrm{B}}\left(1-x_{\mathrm{B}}\right)^{2}-b\left(1-2 x_{\mathrm{B}}+3 x_{\mathrm{B}}^{2}\right)\right.} .
$$

Die Exzeßentropie $\Delta S^{\mathrm{E}}$, die mittlere molare Mischungsenthalpie $\Delta H^{\mathrm{E}}$ sowie die partiellen molaren Mischungsenthalpien berechenen sich für den Redlich-KisterAnsatz nach den folgenden Gleichungen:

$$
\begin{aligned}
& \Delta S^{\mathrm{E}}=-x_{\mathrm{B}}\left(1-x_{\mathrm{B}}\right) b, \\
& \Delta H^{\mathrm{E}}=x_{\mathrm{B}}\left(1-x_{\mathrm{B}}\right)\left(a+B\left(2 x_{\mathrm{B}}-1\right)\right), \\
& H_{\mathrm{A}}^{\mathrm{E}}=x_{\mathrm{B}}^{2}\left(a+B\left(4 x_{\mathrm{B}}-3\right)\right), \\
& H_{\mathrm{B}}^{\mathrm{E}}=\left(1-x_{\mathrm{B}}\right)^{2}\left(a+B\left(4 x_{\mathrm{B}}-1\right)\right) .
\end{aligned}
$$

\section{Wilson-Ansatz}

Aufgrund molekularer Überlegungen leitet Wilson [10] folgenden zweiparametrigen Ausdruck für die freie Exzeßenthalpie $\Delta G^{\mathrm{E}}$ einer binären flüssigen $\mathrm{Mi}$ schung her: $\Delta G^{\mathrm{E}}=R T\left(-x_{\mathrm{A}} \ln \left(x_{\mathrm{A}}+A 1 x_{\mathrm{B}}\right)-x_{\mathrm{B}} \ln \left(x_{\mathrm{B}}+A 2 x_{\mathrm{A}}\right)\right)$.

Der Ansatz nach Wilson stellt eine Modifikation der Flory-Huggins'schen Theorie der athermischen Mischungen dar. Durch die Einführung eines empirischen A-B-Wechselwirkungsgliedes wurde der Anwendungsbereich auch auf Mischungssysteme mit $H^{\mathrm{E}}$ ungleich Null erweitert. Die Herleitung der folgenden, die Liquiduskurven der $\mathrm{B}$-, $\mathrm{AB}$ - und $\mathrm{AB}_{2}$-Addukte beschreibenden Gleichungen sind der Arbeit von Weschke [8] zu entnehmen.

Die Logarithmen der Aktivitätskoeffizienten ergeben sich dabei zu

$\ln f_{\mathrm{A}}=-\ln \left(x_{\mathrm{A}}+A 1 x_{\mathrm{B}}\right)$

$$
+x_{\mathrm{B}}\left[\frac{A 1}{\left(x_{\mathrm{A}}+A 1 x_{\mathrm{B}}\right)}-\frac{A 2}{\left(x_{\mathrm{B}}+A 2 x_{\mathrm{A}}\right)}\right],
$$

$\ln f_{\mathrm{B}}=-\ln \left(x_{\mathrm{B}}+A 2 x_{\mathrm{A}}\right)$

$$
+x_{\mathrm{A}}\left[\frac{A 1}{\left(x_{\mathrm{A}}+A 1 x_{\mathrm{B}}\right)}-\frac{A 2}{\left(x_{\mathrm{B}}+A 2 x_{\mathrm{A}}\right)}\right] \text {. }
$$

Die beiden Parameter $A 1$ und $A 2$ enthalten die Molvolumina der reinen Komponenten und sind mit den charakteristischen Energiedifferenzen $g_{\mathrm{AB}}$ und $g_{\mathrm{BA}}$ folgendermaßen verknüpft:

$$
\begin{aligned}
& A 1=\left(v_{\mathrm{B}} / v_{\mathrm{A}}\right) \exp \left(-g_{\mathrm{AB}} /(R T)\right), \\
& A 2=\left(v_{\mathrm{A}} / v_{\mathrm{B}}\right) \exp \left(-g_{\mathrm{BA}} /(R T)\right) .
\end{aligned}
$$


Die Energiedifferenzen $g_{\mathrm{AB}}$ und $g_{\mathrm{BA}}$ in (13) und (14) beinhalten die Wechselwirkungsenergien $l_{i j}$ zwischen den sich in der Flüssigkeit befindlichen Teilchen:

$$
\begin{aligned}
& g_{\mathrm{AB}}=l_{\mathrm{AB}}-l_{\mathrm{AA}}, \\
& g_{\mathrm{BA}}=l_{\mathrm{BA}}-l_{\mathrm{BB}} .
\end{aligned}
$$

Zur besseren Übersicht seien die folgenden Abkürzungen eingeführt:

$$
\begin{aligned}
A 1 T= & {\left[\frac{\partial A 1}{\partial T}\right]_{p, x}=\frac{\left(v_{\mathrm{B}} / v_{\mathrm{A}}\right)}{R T^{2}} g_{\mathrm{AB}} \exp \left(-g_{\mathrm{AB}} /(R T)\right),(17) } \\
A 2 T= & {\left[\frac{\partial A 2}{\partial T}\right]_{p, x}=\frac{\left(v_{\mathrm{A}} / v_{\mathrm{B}}\right)}{R T^{2}} g_{\mathrm{BA}} \exp \left(-g_{\mathrm{BA}} /(R T)\right),(18) } \\
K 1= & -x_{\mathrm{A}} \ln \left(x_{\mathrm{A}}+A 1 x_{\mathrm{B}}\right)-x_{\mathrm{B}} \ln \left(x_{\mathrm{B}}+A 2 x_{\mathrm{A}}\right), \\
K 1 T= & {\left[\frac{\partial K 1}{\partial T}\right]_{p, x}=-\frac{x_{A} A 1 T x_{\mathrm{B}}}{\left(x_{\mathrm{A}}+\mathrm{A} 1 x_{\mathrm{B}}\right)}-\frac{x_{\mathrm{B}} A 2 T x_{\mathrm{A}}}{\left(x_{\mathrm{B}}+A 2 x_{\mathrm{A}}\right)}, } \\
K 2= & \frac{A 1}{\left(x_{\mathrm{A}}+A 1 x_{\mathrm{B}}\right)}-\frac{A 2}{\left(x_{\mathrm{B}}+A 2 x_{\mathrm{A}}\right)}, \\
K 2 T= & {\left[\frac{\partial K 2}{\partial T}\right]_{p, x}=\frac{A 1 T x_{\mathrm{A}}}{\left(x_{\mathrm{A}}+\mathrm{A} 1 x_{\mathrm{B}}\right)^{2}} } \\
& -\frac{A 2 T x_{\mathrm{B}}}{\left(x_{\mathrm{B}}+A 2 x_{\mathrm{A}}\right)^{2}} .
\end{aligned}
$$

Mit den Aktivitätskoeffizienten für den Wilson-Ansatz (11) und (12) ergeben sich die Gleichungen, welche die Liquiduskurven für den $\mathrm{B}$-, $\mathrm{AB}$-, $\mathrm{AB}_{2}$ - sowie $\mathrm{A}_{2} \mathrm{~B}$ Ast beschreiben:

Liquiduskurve von $\mathrm{B}$ :

$T=\frac{T_{\mathrm{B}}^{\circ} \Delta H_{\mathrm{SB}}}{\Delta H_{\mathrm{SB}}-R T_{\mathrm{B}}^{\circ}\left(\ln x_{\mathrm{B}}-\ln \left(x_{\mathrm{B}}+A 2 x_{\mathrm{A}}\right)-x_{\mathrm{A}} K 2\right)} ;$

Liquiduskurve von $\mathrm{AB}$ :

$T=\frac{T_{\mathrm{AB}}^{\circ} \Delta H_{\mathrm{SAB}}}{\Delta H_{\mathrm{SAB}}-R T_{\mathrm{AB}}^{\circ}\left(\ln \left(x_{\mathrm{A}} x_{\mathrm{B}}\right)-\ln a_{\mathrm{A}}^{\circ}-\ln a_{\mathrm{B}}^{\circ}+\ln f_{\mathrm{A}}+\ln f_{\mathrm{B}}\right)} ;$

Liquiduskurve von $\mathrm{AB}_{2}$ :

$T=\frac{T_{\mathrm{AB}_{2}}^{\circ} \Delta H_{\mathrm{SAB}_{2}}}{\Delta H_{\mathrm{SAB}_{2}}-R T_{\mathrm{AB}_{2}}^{\circ}\left(\ln \left(x_{\mathrm{A}} x_{\mathrm{B}}^{2}\right)-\ln a_{\mathrm{A}}^{\circ}-2 \ln a_{\mathrm{B}}^{\circ}+\ln f_{\mathrm{A}}+2 \ln f_{\mathrm{B}}\right)} ;$

Liquiduskurve von $\mathrm{A}_{2} \mathrm{~B}$ :

$T=\frac{T_{\mathrm{A}_{2} \mathrm{~B}}^{\circ} \Delta H_{\mathrm{SA}_{2} \mathrm{~B}}}{\Delta H_{\mathrm{SA}_{2} \mathrm{~B}}-R T_{\mathrm{A}_{2} \mathrm{~B}}^{\circ}\left(\ln \left(x_{\mathrm{B}} x_{\mathrm{A}}^{2}\right)-\ln a_{\mathrm{B}}^{\circ}-2 \ln a_{\mathrm{A}}^{\circ}+\ln f_{\mathrm{B}}+2 \ln f_{\mathrm{A}}\right)}$.
Für die Exzeßentropie $\Delta S^{\mathrm{E}}$, die mittlere molare Mischungsenthalpie $\Delta H^{\mathrm{E}}$ sowie die partiellen molaren Mischungsenthalpien $H_{i}^{\mathrm{E}}$ ergeben sich die folgenden Gleichungen:

$$
\begin{aligned}
& \Delta S^{\mathrm{E}}=-R(K 1+T K 1 T), \\
& \Delta H^{\mathrm{E}}=-R T^{2} K 1 T, \\
& H_{\mathrm{A}}^{\mathrm{E}}=-R T^{2}\left[\frac{-A 1 T x_{\mathrm{B}}}{\left(x_{\mathrm{A}}+A 1 x_{\mathrm{B}}\right)}+x_{\mathrm{B}} K 2 T\right], \\
& H_{\mathrm{B}}^{\mathrm{E}}=-R T^{2}\left[\frac{-A 2 T x_{\mathrm{A}}}{\left(x_{\mathrm{B}}+A 2 x_{\mathrm{A}}\right)}+x_{\mathrm{A}} K 2 T\right] .
\end{aligned}
$$

\section{NRTL-Ansatz}

Der NRTL-Ansatz von Renon und Prausnitz [12] stellt eine Verknüpfung der „Two Liquid-Theorie“ von Scott [11] mit den „lokalen Volumenbrüchen“ [12] dar.

Die freie Exzeßenthalpie $\Delta G^{\mathrm{E}}$ einer binären flüssigen Mischung hat im NRTL-Ansatz die Form

$\Delta G^{\mathrm{E}}=R T x_{\mathrm{A}} x_{\mathrm{B}}\left[\frac{t 2 G 2}{x_{\mathrm{A}}+x_{\mathrm{B}} G 2}+\frac{t 1 G 1}{x_{\mathrm{B}}+x_{\mathrm{A}} G 1}\right]$,

mit

$t 1=\frac{\left(g_{\mathrm{AB}}-g_{\mathrm{BB}}\right)}{R T}, \quad t 2=\frac{\left(g_{\mathrm{AB}}-g_{\mathrm{AA}}\right)}{R T}$.

$g_{i j}$ ist ein charakteristischer Energieparameter und steht für die Wechselwirkungen der $i$-ten mit der $j$-ten Komponente. Die charakteristischen Energiedifferenzen in (32) und (33) werden in den Parametern $A 3$ und $A 4$ zusammengefaßt:

$$
\begin{array}{ll}
A 3=g_{\mathrm{AB}}-g_{\mathrm{BB}}, & A 4=g_{\mathrm{AB}}-g_{\mathrm{AA}}, \\
G 1=\exp (-\mu t 1), & G 2=\exp (-\mu t 2) .
\end{array}
$$

Der Parameter $\mu$ ist eine temperaturunabhängige, empirische Konstante, welche die Abweichung von der Zufallsverteilung in der Mischung darstellt. Für die Aktivitätskoeffizienten gilt im NRTL-Ansatz

$\ln f_{\mathrm{A}}=x_{\mathrm{B}}^{2}\left[t 2\left(\frac{G 2}{x_{\mathrm{A}}+x_{\mathrm{B}} G 2}\right)^{2}+\frac{t 1 G 1}{\left(x_{\mathrm{B}}+x_{\mathrm{A}} G 1\right)^{2}}\right]$,
$\ln f_{\mathrm{B}}=x_{\mathrm{A}}^{2}\left[t 1\left(\frac{G 1}{x_{\mathrm{B}}+x_{\mathrm{A}} G 1}\right)^{2}+\frac{t 2 G 2}{\left(x_{\mathrm{A}}+x_{\mathrm{B}} G 2\right)^{2}}\right]$.

Unter Verwendung der Aktivitätskoeffizienten aus (38) und (39) lassen sich die Liquiduskurven $\mathrm{B}, \mathrm{AB}$, $\mathrm{AB}_{2}$ und $\mathrm{A}_{2} \mathrm{~B}$ herleiten: 
Liquiduskurve von B:

$T=\frac{T_{\mathrm{B}}^{\circ} \Delta H_{\mathrm{SB}}}{\Delta H_{\mathrm{SB}}-R T_{\mathrm{B}}^{\circ} \ln x_{\mathrm{B}}+\ln f_{\mathrm{B}}} ;$

Liquiduskurve von AB:

$T=\frac{T_{\mathrm{AB}}^{\circ} \Delta H_{\mathrm{SAB}}}{\Delta H_{\mathrm{SAB}}-R T_{\mathrm{AB}}^{\circ}\left(\ln \left(x_{\mathrm{A}} x_{\mathrm{B}}\right)-\ln a_{\mathrm{A}}^{\circ}-\ln a_{\mathrm{B}}^{\circ}+\ln f_{\mathrm{A}}+\ln f_{\mathrm{B}}\right)} ;$

Liquiduskurve von $\mathrm{AB}_{2}$ :

$T=\frac{T_{\mathrm{AB}_{2}}^{\circ} \Delta H_{\mathrm{SAB}_{2}}}{\Delta H_{\mathrm{SAB}_{2}}-R T_{\mathrm{AB}_{2}}^{\circ}\left(\ln \left(x_{\mathrm{A}} x_{\mathrm{B}}^{2}\right)-\ln a_{\mathrm{A}}^{\circ}-2 \ln a_{\mathrm{B}}^{\circ}+\ln f_{\mathrm{A}}+2 \ln f_{\mathrm{B}}\right)} ;$

Liquiduskurve von $\mathrm{A}_{2} \mathrm{~B}$ :

$T=\frac{T_{\mathrm{A}_{2} \mathrm{~B}}^{\circ} \Delta H_{\mathrm{SA}_{2} \mathrm{~B}}}{\Delta H_{\mathrm{SA}_{2} \mathrm{~B}}-R T_{\mathrm{A}_{2} \mathrm{~B}}^{\circ}\left(\ln \left(x_{\mathrm{B}} x_{\mathrm{A}}^{2}\right)-\ln a_{\mathrm{B}}^{\circ}-2 \ln a_{\mathrm{A}}^{\circ}+\ln f_{\mathrm{B}}+2 \ln f_{\mathrm{A}}\right)}$.

Mit den Abkürzungen

$$
\begin{aligned}
& t 1 T=\left[\frac{\partial t 1}{\partial T}\right]_{p, x}, \quad t 2 T=\left[\frac{\partial t 2}{\partial T}\right]_{p, x}, \\
& G 1 T=\left[\frac{\partial G 1}{\partial T}\right]_{p, x}, \quad G 2 T=\left[\frac{\partial G 2}{\partial T}\right]_{p, x}, \\
& K 3=\frac{t 2 G 2}{x_{\mathrm{A}}+x_{\mathrm{B}} G 2}+\frac{t 1 G 1}{x_{\mathrm{B}}+x_{\mathrm{A}} G 1},
\end{aligned}
$$$$
K 3 T=\left[\frac{\partial K 3}{\partial T}\right]_{p, x}
$$$$
=\frac{x_{\mathrm{A}}(t 2 T G 2+G 2 T t 2)+x_{\mathrm{B}} G 2^{2} t 2 T}{\left(x_{\mathrm{A}}+x_{\mathrm{B}} G 2\right)^{2}}
$$$$
+\frac{x_{\mathrm{B}}(t 1 T G 1+G 1 T t 1)+x_{\mathrm{A}} G 1^{2} t 1 T}{\left(x_{\mathrm{B}}+x_{\mathrm{A}} G 1\right)^{2}},
$$$$
K 4=t 2\left[\frac{G 2}{x_{\mathrm{A}}+x_{\mathrm{B}} G 2}\right]^{2}+\frac{t 1 G 1}{\left(x_{\mathrm{B}}+x_{\mathrm{A}} G 1\right)^{2}},
$$$$
K 4 T=\left[\frac{\partial K 4}{\partial T}\right]_{p, x}=\left[\frac{G 2}{x_{\mathrm{A}}+x_{\mathrm{B}} G 2}\right]^{2} t 2 T
$$$$
+\frac{t 22 G 2 T x_{\mathrm{A}} G 2}{\left(x_{\mathrm{A}}+x_{\mathrm{B}} G 2\right)^{3}}
$$$$
+\frac{(t 1 T G 1+t 1 G 1 T)\left(x_{\mathrm{B}}+x_{\mathrm{A}} G 1\right)-2 x_{\mathrm{A}} G 1 T t 1 G 1}{\left(x_{\mathrm{B}}+x_{\mathrm{A}} G 1\right)^{3}},
$$$$
K 5=t 1\left[\frac{G 1}{x_{\mathrm{B}}+x_{\mathrm{A}} G 1}\right]^{2}+\frac{t 2 G 2}{\left(x_{\mathrm{A}}+x_{\mathrm{B}} G 2\right)^{2}},
$$

$$
\begin{aligned}
K 5 T= & {\left[\frac{\partial K 5}{\partial T}\right]_{p, x}=\left[\frac{G 1}{x_{\mathrm{B}}+x_{\mathrm{A}} G 1}\right]^{2} t 1 T } \\
& +\frac{t 12 G 1 T x_{\mathrm{B}} G 1}{\left(x_{\mathrm{B}}+x_{\mathrm{A}} G 1\right)^{3}} \\
+ & \frac{(t 2 T G 2+t 2 G 2 T)\left(x_{\mathrm{A}}+x_{\mathrm{B}} G 2\right)-2 x_{\mathrm{B}} G 2 T t 2 G 2}{\left(x_{\mathrm{A}}+x_{\mathrm{B}} G 2\right)^{3}},
\end{aligned}
$$

lassen sich die Exzeßgrößen darstellen:

$$
\begin{aligned}
& \Delta S^{\mathrm{E}}=-R x_{\mathrm{A}} x_{\mathrm{B}}(K 3+T K 3 T), \\
& \Delta H^{\mathrm{E}}=-R T^{2} x_{\mathrm{A}} x_{\mathrm{B}} K 3 T, \\
& H_{\mathrm{A}}^{\mathrm{E}}=-R T^{2} x_{\mathrm{B}}^{2} K 4 T, \\
& H_{\mathrm{B}}^{\mathrm{E}}=-R T^{2} x_{\mathrm{A}}^{2} K 5 T .
\end{aligned}
$$

Eine ausführliche Ableitung der Gleichungen zur Beschreibung der Liquiduskurven $\mathrm{B}, \mathrm{AB}, \mathrm{AB}_{2}$ für den Wilson- und den NRTL-Ansatz findet sich bei Weschke [8].

\section{Ausgleichsrechnung}

Die unbekannten Parametersätze für die den nichtidealen Fall beschreibenden Liquiduskurven wurden mit dem Minimierungsverfahren nach Nelder-Mead [13] so ermittelt, daß die jeweils zutreffenden Funktionen optimal den entsprechenden Meßpunkten aus den Schmelzdiagrammen der Methyltrichlor- und Dimethyldichlorsilane mit Lutidin [3-5] angepaßt waren.

Neben der Schmelzenthalpie, sowie bei inkongruent schmelzenden Verbindungen der Schmelztemperatur der jeweils betrachteten Verbindungen finden im Ansatz nach Redlich und Kister die Parameter $a, b, B$ [2], im Ansatz nach Wilson $A 1$ und $A 2$ sowie im NRTLAnsatz die Parameter $\mu, A 3$ und $A 4$ [8] Verwendung. Die Parametersätze der die Liquiduskurven beschreibenden Gleichungen wurden variiert und das gemeinsame Minimum nach der Methode der kleinsten Fehlerquadrate aufgesucht [2]. Nach diesem Verfahren wurden die Parametersätze des jeweiligen Ansatzes für die verschiedenen Berechnungsmethoden -1 . Berechnung des Amin-Astes (B-Ast) zusammen mit dem Kurvenast der sich anschließenden Verbindung $\left(\Delta H_{\mathrm{SB}}\right.$ wurde fest vorgegeben, $\Delta H_{\mathrm{S}}$ der Verbindung wurde variiert), 2. Berechnung des B-Astes mit $\Delta H_{\mathrm{SB}}$ (variabel), 3. Berechnung des B-Astes mit $\Delta H_{\mathrm{SB}}$ (fest vorgegeben) - ermittelt. 
Näherungsweise Berechnung der Dissoziationsenthalpie

Die Schmelzenthalpie einer Verbindung setzt sich aus dem Teil der Schmelzenthalpie, der dem Übergang vom festen in den flüssigen Aggregatzustand ohne Dissoziation der Verbindung zuzuordnen ist, und der Dissoziationsenthalpie unter der Voraussetzung vollständiger Dissoziation zusammen:

$$
\Delta H_{\mathrm{S}}=\Delta H_{\mathrm{S}}^{\circ}+\Delta H_{\mathrm{D}} .
$$

Da der Anteil der Dissoziationsenthalpie nicht einfach durch direkte Messung mit einem Thermoanalysegerät zugänglich ist, muß er durch indirekte Methoden bestimmt werden. Unter der Annahme von Näherungen kann man dazu folgende Kreisprozesse für die Addukte $\mathrm{AB}, \mathrm{AB}_{2}$ [2] und $\mathrm{A}_{2} \mathrm{~B}$ benutzen:

AB-Addukt:

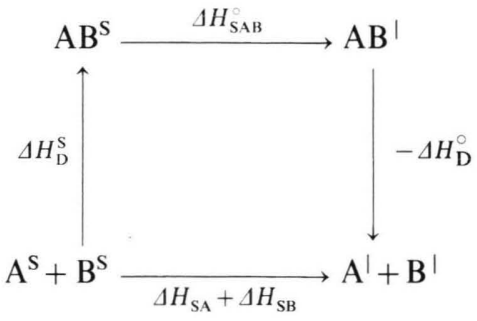

$\mathrm{AB}_{2}$-Addukt:

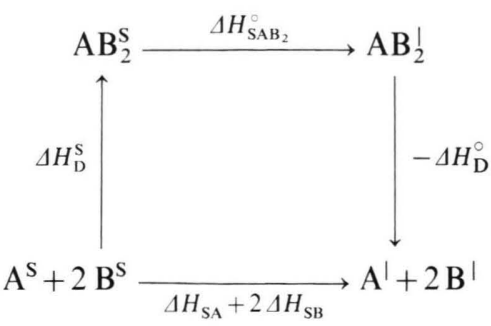

$\mathrm{A}_{2}$ B-Addukt:

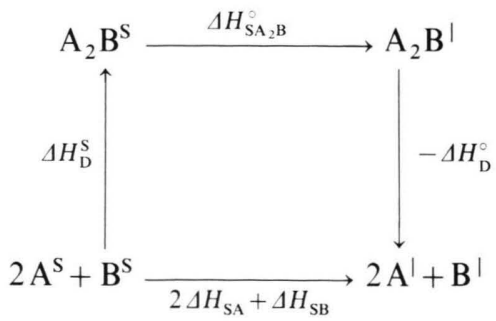

Setzt man nun näherungsweise die Dissoziationsenthalpie in den beiden koexistenten Phasen gleich, so läßt sich die Schmelzenthalpie (ohne Dissoziationsenthalpie) der Prozesse nach

$$
\begin{array}{ll}
\Delta H_{\mathrm{SAB}}^{\circ}=\Delta H_{\mathrm{SA}}+\Delta H_{\mathrm{SB}} & \text { (für ein 1:1-Addukt), } \\
\Delta H_{\mathrm{SAB}_{2}}^{\circ}=\Delta H_{\mathrm{SA}}+2 \Delta H_{\mathrm{SB}} & \text { (für ein 1:2-Addukt), } \\
\Delta H_{\mathrm{SA}_{2} \mathrm{~B}}^{\circ}=2 \Delta H_{\mathrm{SA}}+\Delta H_{\mathrm{SB}} & \text { (für ein 2:1-Addukt) }
\end{array}
$$

berechnen. Die Dissoziationsenthalpien können damit aus den folgenden Gleichungen berechnet werden:

$$
\begin{aligned}
\Delta H_{\mathrm{DAB}}^{\circ} & =-\Delta H_{\mathrm{RAB}}^{\circ} \quad \text { (für ein 1:1-Addukt) } \\
& =\Delta H_{\mathrm{SAB}}-\Delta H_{\mathrm{SA}}-\Delta H_{\mathrm{SB}}, \\
\Delta H_{\mathrm{DAB}_{2}}^{\circ} & =-\Delta H_{\mathrm{RAB}_{2}}^{\circ} \quad \text { (für ein 1:2-Addukt) } \\
& =\Delta H_{\mathrm{SAB}_{2}}-\Delta H_{\mathrm{SA}}-2 \Delta H_{\mathrm{SB}}, \\
\Delta H_{\mathrm{DA}_{2} \mathrm{~B}}^{\circ} & =-\Delta H_{\mathrm{RA}_{2} \mathrm{~B}}^{\circ} \quad \text { (für ein 2:1-Addukt) } \\
& =\Delta H_{\mathrm{SA}_{2} \mathrm{~B}}-2 \Delta H_{\mathrm{SA}}-\Delta H_{\mathrm{SB}},
\end{aligned}
$$

$\Delta H_{\mathrm{D}}^{\circ}=$ Dissoziationsenthalpie,

$\Delta H_{\mathrm{R}}^{\circ}=$ Reaktionsenthalpie.

Zur Berechnung der Dissoziationsenthalpie der Methyltrichlor- bzw. Dimethyldichlorsilan/LutidinAddukte wurden die mit Hilfe einer DSC 30 der Firma Mettler gemessenen Schmelzenthalpien der Reinsubstanzen sowie der Verbindungen verwendet [5].

\section{Ergebnisse und Diskussion}

Mit Hilfe einer DSC 30 der Firma Mettler wurden die Schmelzenthalpien eines Teils der für die Systeme, bestehend aus Methyltrichlor- bzw. Dimethyldichlorsilan mit Lutidin, nachgewiesenen Verbindungen gemessen [5]. Dabei zeigte sich, daß die Schmelzenthalpien für die $\mathrm{MeSiCl}_{3} /$ Lutidin-Addukte bis auf eine Ausnahme (2.3-Lutidin) größer als die der $\mathrm{Me}_{2} \mathrm{SiCl}_{2} /$ Lutidin-Verbindungen waren. Da die gemessenen Schmelzenthalpien einen unbekannten Anteil an Kristallisationsenthalpie enthalten, erschien es sinnvoll, die Dissoziationsenthalpien näherungsweise zu berechnen (Tab.1). Dabei ergibt sich für die 1:2-Verbindungen eine deutliche Abhängigkeit der Dissoziationsenthalpie vom Silan. $\mathrm{MeSiCl}_{3}$ weist dabei mit 3.4- und 3.5-Lutidin eine stark positive Dissoziationsenthalpie (negative Reaktionsenthalpie) auf, was auf Wechselwirkungen zwischen den Reaktionspartnern in der flüssigen Phase schließen läßt. Für die übrigen 1:2Addukte ist die Dissoziationsenthalpie nahezu Null bzw. negativ. Da die Dissoziationsenthalpie als ein 
Tab. 1. Gemessene und errechnete Schmelzenthalpien für die Systeme Methyltrichlor- bzw. Dimethyldichlorsilan mit Lutidin sowie die berechneten Dissoziationsenthalpien für diese Verbindungen.

\begin{tabular}{|c|c|c|c|c|c|}
\hline System & $\begin{array}{l}\Delta H_{\mathrm{S}} \text { (gemessen) } \\
{[\mathrm{kJ} / \mathrm{mol}]}\end{array}$ & $\begin{array}{l}\Delta H_{\mathrm{D}}^{\circ} \\
{[\mathrm{kJ} / \mathrm{mol}]}\end{array}$ & $\begin{array}{l}\text { Redlich-Kister } \\
{[\mathrm{kJ} / \mathrm{mol}]}\end{array}$ & $\begin{array}{l}\text { Wilson } \\
{[\mathrm{kJ} / \mathrm{mol}]}\end{array}$ & $\begin{array}{l}\text { NRTL } \\
{[\mathrm{kJ} / \mathrm{mol}]}\end{array}$ \\
\hline $\mathrm{MeSiCl}_{3} \cdot(2.3 \text {-Lutidin })_{2}$ & 28,4 & $-3,9$ & 12,2 & 1,7 & 12,3 \\
\hline $\mathrm{MeSiCl}_{3} \cdot 2.4$-Lutidin & 16,8 & 0,4 & 111,9 & 46,0 & 89,5 \\
\hline $\mathrm{MeSiCl}_{3} \cdot(2.5 \text {-Lutidin })_{2}$ & 31,4 & $-3,3$ & 17,3 & 17,5 & 14,2 \\
\hline $\mathrm{MeSiCl}_{3} \cdot(2.6 \text {-Lutidin })_{2}$ & 30,3 & $-3,2$ & 16,5 & 15,1 & 15,1 \\
\hline$\left(\mathrm{MeSiCl}_{3}\right)_{2} \cdot 3.4$-Lutidin & 31,4 & 2,2 & 11,6 & 29,9 & 38,1 \\
\hline $\mathrm{MeSiCl}_{3} \cdot(\text { 3.4-Lutidin })_{2}$ & 66,8 & 33,9 & 270,4 & 90,0 & 152,8 \\
\hline$\left(\mathrm{MeSiCl}_{3}\right)_{2} \cdot 3.5$-Lutidin & 29,3 & $-0,4$ & 57,4 & 40,7 & 35,5 \\
\hline $\mathrm{MeSiCl}_{3} \cdot(3.5 \text {-Lutidin })_{2}$ & 75,1 & 41,4 & 103,4 & 172,5 & 125,0 \\
\hline $\mathrm{Me}_{2} \mathrm{SiCl}_{2} \cdot(2.3 \text {-Lutidin })_{2}$ & 35,1 & 3,1 & 11,7 & 14,2 & 16,9 \\
\hline $\mathrm{Me}_{2} \mathrm{SiCl}_{2} \cdot 2.4$-Lutidin & 5,6 & $-10,6$ & 8,4 & 4,5 & 4,3 \\
\hline $\mathrm{Me}_{2} \mathrm{SiCl}_{2} \cdot 2.5$-Lutidin & 17,0 & $-4,5$ & & & \\
\hline $\mathrm{Me}_{2} \mathrm{SiCl}_{2} \cdot(2.5 \text {-Lutidin })_{2}$ & 22,7 & $-11,8$ & 28,3 & 20,9 & 19,1 \\
\hline $\mathrm{Me}_{2} \mathrm{SiCl}_{2} \cdot$ 2.6-Lutidin & 6,0 & $-14,8$ & & & \\
\hline $\begin{array}{l}\mathrm{Me}_{2} \mathrm{SiCl}_{2} \cdot(2.6 \text {-Lutidin })_{2} \\
\mathrm{Me} \mathrm{SiCl}_{2} \cdot 3 \text {-Lutidin }\end{array}$ & 23,8 & 9,5 & 9,7 & 11,8 & 11,6 \\
\hline $\begin{array}{l}\mathrm{Me}_{2} \mathrm{SiCl}_{2} \cdot 3.5 \text {-Lutidin } \\
\mathrm{Me}_{2} \mathrm{SiCl}_{2} \cdot(3.5 \text {-Lutidin })_{2}\end{array}$ & $\begin{array}{l}11,5 \\
28,6\end{array}$ & $\begin{array}{l}-9,5 \\
-5,0\end{array}$ & 3,8 & 9,6 & 9,5 \\
\hline
\end{tabular}

Maß für die Lewis-Säure-Base-Reaktion der Moleküle angesehen werden kann, zeigt sich, daß die Bildung der Addukte bis auf die Verbindungen $\mathrm{MeSiCl}_{3} \cdot(3.5 \text {-Lutidin) })_{2}$ und $\mathrm{MeSiCl}_{3} \cdot(3.4 \text {-Lutidin) })_{2}$ überwiegend durch die Kristallisationsenthalpie begünstigt wird, also weniger auf eine spezifische LewisSäure-Base-Wechselwirkung zurückzuführen ist.

Diese Aussage wird dadurch bekräftigt, daß keine Korrelation zwischen dem $\mathrm{pK}_{\mathrm{A}}$-Wert der Lutidine (gegenüber Protonen [14]) und der Dissoziationsenthalpie, mit Ausnahme von 3.5-Lutidin als stärkster Base unter den Lutidinen, besteht.

Mit den mittels Minimierungsverfahren nach Nelder-Mead für die Ansätze nach Redlich und Kister, Wilson sowie für den NRTL-Ansatz ermittelten Parametersätzen werden die Kurvenverläufe der Schmelzdiagramme, bestehend aus Methyltrichlorbzw. Dimethyldichlorsilan mit Lutidin gut wiedergegeben [15]. Wie jedoch bereits von Weschke [8] beschrieben, ergeben sich auch bei diesen Systemen in der Regel unterschiedliche numerische Werte für die Schmelzenthalpien in den drei Ansätzen (Tab. 1). Der Vergleich dieser errechneten Schmelzenthalpien mit den gemessenen $\Delta H_{\mathrm{S}}$-Werten zeigt nur in einigen Fällen für den Wilson- und den NRTL-Ansatz eine Abweichung unter $25 \%$ vom gemessenen $\Delta H_{\mathrm{s}}$-Wert. Mit dem Ansatz nach Redlich und Kister konnten keine befriedigenden $\Delta H$-Werte errechnet werden.

Da diese Ansätze nur bedingt zur Berechnung von komplexeren Schmelzdiagrammen dieser Substanzklasse geeignet erschienen, wurden sie zur Berechnung
Tab. 2. Gemessene und errechnete Schmelzenthalpien für die B-Äste in den Systemen Methyltrichlorsilan mit Lutidin.

\begin{tabular}{lcccc}
\hline System & $\Delta H_{\mathrm{S}}(\mathrm{gem})$. & $\begin{array}{l}\text { Redlich- } \\
\text { Kister } \\
{[\mathrm{kJ} / \mathrm{mol}]}\end{array}$ & $\begin{array}{l}\text { Wilson } \\
{[\mathrm{kJ} / \mathrm{mol}]}\end{array}$ & NRTL \\
& $[\mathrm{kJ} / \mathrm{mol}] \mathrm{mol}]$ \\
\hline 2.3-Lutidin & 11,9 & 4,0 & 20,5 & 11,4 \\
2.4-Lutidin & 7,8 & 12,0 & 8,9 & 8,9 \\
2.5-Lutidin & 13,6 & 7,2 & 6,9 & 6,7 \\
2.6-Lutidin & 12,5 & 11,5 & 12,1 & 9,7 \\
\hline
\end{tabular}

einzelner Abschnitte der Phasendiagramme, speziell der Amin-Äste, herangezogen. Wie schon im zuvor beschriebenen Fall der Berechnung des B-Astes mit der sich anschließenden Liquiduskurve der Verbindungsbildung (als $\Delta H_{\mathrm{SB}}$ wurden hier die gemessenen Werte eingesetzt), konnten die Schmelzenthalpien der Amine mit dem Ansatz nach Redlich und Kister bis auf eine Ausnahme nicht befriedigend berechnet werden; für die Schmelzenthalpie von 3.4-Lutidin in dem System 3.4-Lutidin/Dimethyldichlorsilan wurde sogar eine physikalisch unsinnige negative Schmelzenthalpie errechnet (Tab. 2 und 3). Dagegen lieferten der Wilson- und der NRTL-Ansatz in jeweils $50 \%$ der Fälle eine mit den gemessenen $\Delta H_{\mathrm{SB}}$-Werten gut übereinstimmende Schmelzenthalpie. Beide Ansätze lieferten in bezug auf die errechneten Schmelzenthalpien zumindest in der Tendenz der numerischen Werte das gleiche Ergebnis. Daraus ergibt sich, daß die Amin- Äste der Phasendiagramme in der Regel von beiden Ansätzen gemeinsam besser oder schlechter beschrieben 
Tab. 3. Gemessene und errechnete Schmelzenthalpien für die B-Äste in den Systemen Dimethyldichlorsilan mit Lutidin.

\begin{tabular}{lcccc}
\hline System & $\Delta H_{\mathrm{S}}$ (gem.) & $\begin{array}{l}\text { Redlich- } \\
\text { Kister } \\
{[\mathrm{kJ} / \mathrm{mol}]}\end{array}$ & {$[\mathrm{kJ} / \mathrm{mol}]$} & {$[\mathrm{kJ} / \mathrm{mol}]$} \\
\hline$[\mathrm{kJ} / \mathrm{mol}]$ & & 21,6 & 25,0 & 24,8 \\
2.3-Lutidin & 11,9 & 2,4 & 8,2 & 10,7 \\
2.4-Lutidin & 7,8 & 2,9 & 5,0 & 7,3 \\
2.5-Lutidin & 13,6 & 7,9 & 10,1 & 9,6 \\
2.6-Lutidin & 12,5 & $(-1,3)$ & 38,8 & 35,0 \\
3.4-Lutidin & 12,1 & 7,7 & 15,6 & 15,6 \\
3.5-Lutidin & 12,6 & & & \\
\hline
\end{tabular}

werden. Für die Systeme, bei denen die Übereinstimmung der gemessenen mit den errechneten Schmelzenthalpien für das Amin gut war, zeigten auch die aus den jeweiligen Parametersätzen errechneten Exzeßgrößen nahezu die gleichen Kurvenverläufe.

Das Einsetzen der mit einer DSC gemessenen Schmelzenthalpien in die verwendeten Ansätze zur Berechnung der Amin-Äste führte neben der Verringerung der Variationsparameter zu einer höheren Sicherheit beim Auffinden des Parametersatzes mit der kleinsten Fehlerquadratsumme für verschiedene Startparametersätze. Die Wahrscheinlichkeit, durch ungünstige Wahl der Startparameter in ein Nebenminimum zu gelangen, wird dadurch deutlich ge-

[1] K. Hensen u. R. Busch, Z. Naturforsch. 37 b, 1174 (1982).

[2] K. Hensen u. J. Gaede, Z. Naturforsch. 42a, 341 (1987).

[3] K. Hensen u. M. Dräbing, Z. Naturforsch. 44b, 63 (1989).

[4] K. Hensen u. M. Dräbing, Z. Naturforsch. 45b, 99 (1990).

[5] K. Hensen u. M. Dräbing, Z. Naturforsch. 44a, 1172 (1989).

[6] W. Heberer, Dissertation, Frankfurt am Main 1988

[7] J. Grebner, Diplomarbeit, Frankfurt am Main 1988.

[8] M. Weschke, Dissertation, Frankfurt am Main 1988. ringer. Bei den zuvor beschriebenen Berechnungen wurde diese Fehlerquelle durch eine breite Variation in der Wahl der Startparameter weitestgehend ausgeschlossen. Über die Güte der Ergebnisse dieser Rechnung kann jedoch erst durch die Gegenüberstellung der errechneten mit den gemessenen Exzeßgrößen eine Aussage gemacht werden.

Vergleicht man die Exzeßfunktionen für die einzelnen Berechnungsmethoden (1. Berechnung des BAstes mit der Liquiduskurve der sich anschließenden Verbindung, $\Delta H_{\mathrm{SB}}$ (vorgegeben), $\Delta H_{\mathrm{S}}$ der Verbindung wurde variiert; 2 . des B-Astes, $\Delta H_{\mathrm{SB}}$ (variiert); 3 . des B-Astes, $\Delta H_{\mathrm{SB}}$ (vorgegeben)) der verwendeten Ansätze im Molenbruchbereich $0<x<0,35$, dann zeigen sich für einen Ansatz oft sehr unterschiedliche Kurvenverläufe. Zusammenfassend ergibt sich, daß die auf molekularen Überlegungen beruhenden Ansätze (Wilson, NRTL) in bezug auf die errechnete Schmelzenthalpie bessere Ergebnisse liefern, als der aus einer Potenzreihe resultierende Redlich-Kister-Ansatz. In Verbindung mit den Ergebnissen bei der Berechnung der Dissoziationsenthalpien, speziell der hohen Dissoziationsenthalpien bei den Systemen Trichlormethylsilan mit 3.4- und 3.5-Lutidin, sollte man bei der Betrachtung der numerischen Ergebnisse die zur Aufstellung der Gleichungen verwendeten Vereinfachungen nicht vergessen.

[9] O. Redlich u. A. T. Kister, Ind. Eng. Chem. 40, 345 (1948).

[10] G. M. Wilson, J. Amer. Chem. Soc. 86, 127 (1964).

[11] R. L. Scott, J. Amer. Phys. 25, 193 (1956).

[12] J. M. Prausnitz, Molecular Thermodynamics of FluidPhase Equilibria, Prentice Hall, Inc., Englewood Cliffs, New Jersey.

[13] J. A. Nelder u. R. Mead, The Computer Journal 7, 308 (1965).

[14] R. J. L. Andon, J. D. Cox u. E. F. G. Herington, Trans. Faraday Soc. 50, 918 (1954).

[15] M. Dräbing, Dissertation, Frankfurt am Main 1989. 